\title{
Differences between Trunk Heat Patterns Shown by Complete and Incomplete Spinal Cord Injured Veterans *
}

\author{
Richard A. Sherman, M.S., Ph.D., ${ }^{1}$ Jeffrey L. Ernst, Ph.D. ${ }^{2}$ and Janusz \\ Markowski, M.D. ${ }^{3}$ \\ ${ }^{1}$ Chief, Psychophysiology Service Department of Clinical Investigation, DD \\ Eisenhower Army Medical Center, Fort Gordon, Georgia, U.S.A. ${ }^{2}$ Psychology \\ Service Veterans Administration Medical Center Augusta, Georgia, U.S.A. \\ ${ }^{3}$ Chief, Spinal Cord Injury Unit Veterans Administration Medical Center Augusta, \\ Georgia, U.S.A.
}

\section{Summary}

Thermograms showing heat patterns in the trunk of the body were taken of ten intact subjects, seven subjects with incomplete spinal cord injuries (SCI), and fifteen subjects diagnosed as having complete SCIs. Fourteen of the subjects with complete SCIs had a thermal demarcation line across the trunk. This line represented a temperature gradient of one to 2.5 degrees Celsius between a relatively warm upper level where sensations were normal and a relatively cool lower level in which sensations were either absent or different from pre-injury sensations. The transition zone was narrow and sharp for ten of the complete SCI subjects but was wide and gradual for the other five complete SCI subjects. The shape and width of the thermal transition zone corresponded to the location and degree of abruptness with which sensations changed from normal to abnormal. One of the subjects diagnosed as having a complete SCI failed to show a significant transition zone. The temperature difference between the upper and lower portions of his trunk was less than one degree Celsius. None of the intact or incomplete SCI subjects produced the transition zone. Only one incomplete SCI subject showed even a minimal difference in temperature between the normal sensation and abnormal sensation levels. A panel blind to the subjects' conditions was unable to differentiate between intact and incomplete SCI subjects but was able to sort complete from incomplete SCI subjects in all but the case with an indistinct transition zone.

Key words: Spinal cord injuries; Thermography patterns

\section{Introduction}

A major problem in the evaluation of spinal cord injured patients is the rapid, non-invasive determination of whether the injury is complete. Careful neuro-

^ Portions of this work were presented at the 1986 annual meeting of the American Spinal Injury Association held in San Francisco, California.

Correspondence and reprint requests to: Major Richard A. Sherman, Ph.D., Department of Clinical Investigation, Fitzsimons Army Medical Center, Aurora, CO 80045. 
logical evaluations such as those detailed in our recent report on relationships between heat patterns produced by SCI veterans and location of chronic phantom body pain (Sherman, et al., 1986) are typical of the inexact way most patients are diagnosed. The continuing lack of a well accepted, objective method for differentiating complete from incomplete SCI patients was discussed extensively by participants at both the 1985 meeting of the American Paraplegia Society (Las Vegas, Nevada) and the 1986 meeting of the American Spinal Injury Association (San Francisco, California).

While carrying out the above study of phantom pain among SCI veterans, we noticed that those patients who were diagnosed as having complete SCIs appeared to have different thermographic patterns on their chests and backs than those diagnosed as having incomplete SCIs. This report presents the results from the first 22 SCI patients for whom we had sufficient data to carry out formal evaluations of chest or back thermographic patterns.

People with spinal cord injuries frequently have difficulties with a variety of aspects of thermoregulation. The inability to shiver, the lack of vasoconstriction to conserve heat, and the inability to perspire for heat dissipation are frequent sequelae of SCI (Trieschmann 1980). We are not the first workers to note differences between intact and SCI subjects in heat emanating from the trunk. Guttmann (1976) reviewed the alterations in sweating induced by SCI as recorded through the use of liquid crystal techniques. Normell (1974) reviewed the literature and carried out a methodological study on impaired cutaneous thermoregulatory vasomotor responses using infrared thermographic techniques. However, neither author reported work which compared thermographic patterns produced by complete and incomplete SCI subjects. Both of these authors reviewed the literature on thermoregulatory mechanisms among people with SCIs.

\section{Patients and methods}

The ten intact subjects were drawn from the staff at a large medical centre. The spinal cord injured subjects were all male veteran patients at a US Veterans Administration Medical Center SCI unit. Fifteen were diagnosed as having complete injuries and seven were diagnosed as being incomplete. The standard clinical evaluation we used was described elsewhere (Sherman et al., 1986b). Potential participants were rejected if there was any doubt of the diagnosis. We could be certain which potential subjects were incomplete but, of course, had the same difficulty all other groups have determining whether subjects were actually complete so were conservative in selecting cases diagnosed as complete. No recent injuries were included to preclude the likelihood of later return of function. No subjects were used in this study who had pain in the upper trunk in order to preclude the possibility that changes due to pain would confound changes due to the type of injury. The SCI subjects' injuries are described in the Table.

Each subject answered a series of demographic and historical questions concerning his injury. This included a detailed description of sensations perceived as emanating from below the level of injury. Information about sensations in the transition zone above which sensations were normal and below which sensa- 
Table Characteristics of Spinal Cord Injured Subjects

\begin{tabular}{|c|c|c|c|c|c|c|}
\hline Sub & Level & $\begin{array}{l}\text { Trunk Temp. } \\
\text { Chng. Sharp or } \\
\text { Gradual }\end{array}$ & $\begin{array}{l}\text { Line Across } \\
\text { Entire Trunk }\end{array}$ & $\begin{array}{l}\text { Degrees C } \\
\text { Change } \\
\text { Top to } \\
\text { Bottom }\end{array}$ & View & $\begin{array}{l}{ }_{0} \text { Of Panel } \\
\text { Correctly } \\
\text { Sorted pt. }\end{array}$ \\
\hline \multicolumn{7}{|c|}{ A. Complete Spinal Cord Injuries } \\
\hline 1 & $\mathrm{C} 5 / 6$ & sharp & yes & 1.5 Cels & chest & $\mathrm{N} / \mathrm{A}$ \\
\hline 2 & $\mathrm{C} 6 / 7 / \mathrm{T} 11 / 12$ & sharp & yes & $2 \cdot 5$ & chest & $100^{\circ}{ }_{0}$ \\
\hline 3 & $\mathrm{C} 6$ & gradual & yes & $0 \cdot 5$ & back & $30^{\circ}{ }_{0}^{\circ}$ \\
\hline 4 & $\mathrm{~T} 7$ & gharp & yes & $1 \cdot 0$ & back & $90^{\circ}{ }_{0}^{\circ}$ \\
\hline 5 & $\mathrm{C} 7$ & gradual & almost & $1 \cdot 0$ & chest & $90^{\circ}$ \\
\hline 6 & $\mathrm{~T} 11 / 12 / \mathrm{L} 3$ & gradual & almost & $2 \cdot 5$ & chest & $\mathrm{N} / \mathrm{A}$ \\
\hline 8 & $\mathrm{~T} 10$ & sharp & yes & $1 \cdot 0$ & back & $\mathrm{N} / \mathrm{A}$ \\
\hline 10 & $\mathrm{~T} 11$ & gradual & yes & $1 \cdot 0$ & back & $\mathrm{N} / \mathrm{A}$ \\
\hline 11 & $\mathrm{C} 5 / 6$ & sharp & yes & $1 \cdot 0$ & back & $\mathrm{N} / \mathrm{A}$ \\
\hline 12 & $\mathrm{C} 5 / 6$ & sharp & yes & 1.5 & chest & $\mathrm{N} / \mathrm{A}$ \\
\hline 13 & $\mathrm{~T} 4$ & sharp & yes & $1 \cdot 0$ & chest & $\mathrm{N} / \mathrm{A}$ \\
\hline 14 & $\mathrm{~T} 10$ & sharp & yes & 1.5 & chest & $\mathrm{N} / \mathrm{A}$ \\
\hline 15 & $\mathrm{C} 6 / 7$ & sharp & yes & $1 \cdot 5$ & chest & $90^{\circ}{ }_{0}$ \\
\hline 16 & $\mathrm{~T} 7$ & fairly sharp & yes & $2 \cdot 5$ & chest & $N / A$ \\
\hline 17 & T9 & graudal & yes & 1.5 & $\begin{array}{l}\text { back \& } \\
\text { chest }\end{array}$ & $\mathrm{N} / \mathrm{A}$ \\
\hline \multicolumn{7}{|c|}{ B. Incomplete Spinal Cord Injuries } \\
\hline 18 & $\mathrm{C} 5 / 6$ & gradual & no & $0 \cdot 5$ & back & $100^{\circ}{ }_{0}$ \\
\hline 19 & $\mathrm{C} 3$ & very gradual & no & $0 \cdot 0$ & back & $100^{\circ} 0$ \\
\hline 20 & L1 & very gradual & none & 0.5 & chest & $90^{\circ}{ }_{0}$ \\
\hline 21 & $\mathrm{~T} 12 / 13$ & very gradual & none & $0 \cdot 5$ & chest & $100^{\circ}{ }_{0}^{\circ}$ \\
\hline 22 & $\mathrm{~T} 12$ & very gradual & none & $0 \cdot 5$ & chest & $100^{\circ}{ }_{0}$ \\
\hline 23 & T9 & very gradual & no & $1 \cdot 0$ & chest & $100^{\circ}{ }_{0}^{\circ}$ \\
\hline 24 & $\mathrm{C} 5 / 6$ & very gradual & no & 0.5 & chest & $50^{\circ}$ \\
\hline
\end{tabular}

tions were either absent or different from pre-injury 'normal' feelings were also elicited. Each subject filled in (or guided our filling in) an outline diagram of the body in which the transition zone was demarcated as being sharp or graudal.

All subjects were thermographed while sitting or lying in bed. The entire trunk was exposed 15 minutes prior to recording to permit an adequate equilibration period for body temperature stabilization. Room temperature and humidity were not recorded on a daily basis. However, the SCI unit is in a new facility which is designed to maintain these factors within narrow, constant limits due to the thermoregulatory problems experienced by SCI patients which were discussed above. Thus, all recordings were done on subjects living within the same, consistent environment. Near surface blood flow patterns were recorded using either an Inframetrics model 520 or a model 535 videothermograph to detect surface temperature patterns. These instruments were capable of resolving temperature differences of $0 \cdot 1$ degrees Celsius and were sensitive to the heat created by blood flow patterns at least $1.5 \mathrm{~cm}$. deep. Thus, all heat sources in a structure as thin as a hand would be visible but only a diffuse reflection of heat sources deep in the body cavity would be visible in a structure as thick as the trunk. The device produces either grey tone or colour images on a television screen. When colour is used, each colour in a spectrum reflects a given range of temperature. Sensitivity can be adjusted so that each colour covers a range of between 0.1 and 20 degrees. We used a sensitivity of 0.5 degrees per colour because we and others (Harway, 1986) have found that normal variation between extremities is more than half a degree but less than one degree Celsius. Thus, 
we used a sensitivity which would minimise formation of insignificant patterns but would not decrease our ability to resolve real differences. The model 535 thermograph has an internal temperature reference. When the model 520 was used, an AGA model 23 temperature source provided a consistent temperature reference, so that actual temperature levels could be objectively measured. The video images were recorded on polaroid photographs for later analysis.

\section{Data evaluation}

Evaluation of thermographs. Thermograms of the chest and, when available, the back were evaluated for differences in pattern of temperature and in the actual temperature above, in, and below the transition line. Actual temperature was determined as discussed above by comparing the colour of the reference to colours of the skin area of interest. As each colour covered a half degree centigrade span, readings were reliable within half a degree. Since half a degree is also the amount of normal variability, none of our data was considered to include reliable differences unless differences in temperature of at least one degree were present. This method of determining temperature differences and the conservative approach to evaluating colour differences provide objective, reliable data. However, thermography is not yet an accepted, standard technique in common clinical use. Moreover, we are not aware of any studies in which thermograms were evaluated by raters who were blind to the problem they were evaluating. In view of the lack of substantiation for the technique itself, we felt that even the highly objective results of this study would be questionable unless we provided evidence that independent raters would come to the same conclusions we did about relationship between temperature patterns and pain. The use of a blind panel is especially appropriate because the difference between complete and incomplete SCI patients was being partially determined from examination of photographs to determine the presence or absence of a line. This could easily be considered to be a subjective judgement liable to all of the well established pitfalls of investigator bias. As part of a larger study (Sherman et al., 1986a), a team of five PhD level scientists and five orthopedic surgeons blindly reviewed thermograms of SCI veterans. None of the raters were familiar with the subjects pictured and all relevant notations were removed from the thermograms. The raters were asked to sort the thermograms into complete SCI, incomplete SCI, or no SCI sets. Thermograms of five intact people were mixed with those of incomplete SCI patients when raters were asked to sort intact from incomplete SCI people.

Statistical associations. The number of degrees difference between the areas above and below the transition zone between normal and abnormal sensations was established by counting the number of half degree colours between the two areas. The resulting numbers from each group were compared using a Student's ' $t$ ' test.

The results of the blind study were analysed by rank ordering the correct answers and the rater's responses. Correlations between the ranks were determined using Spearman's formula except where a tie occurred. When there were ties, we split the ranks between the members of the tie and used Pearson's 


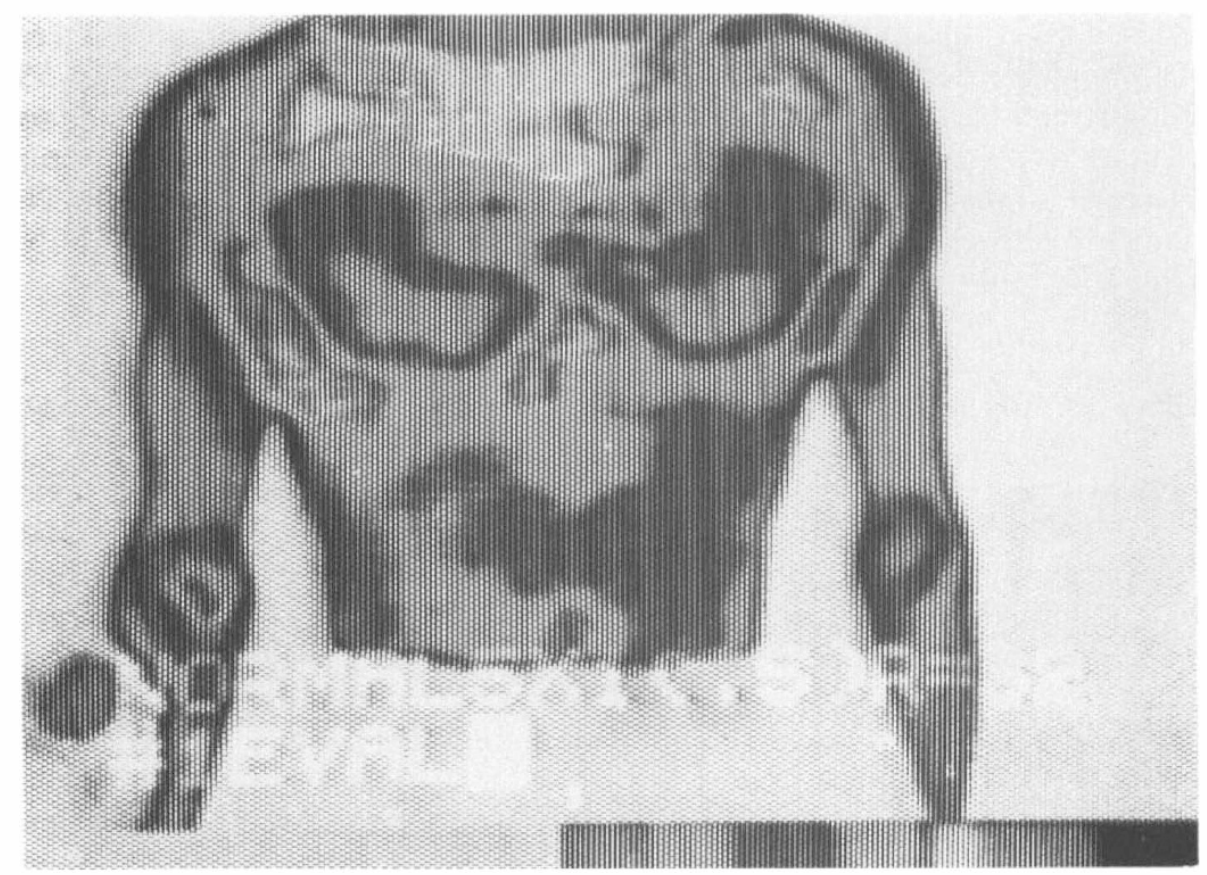

Figure 1 Is a typical chest thermogram of a normal subject. The illustration is a greytone reproduction of a colour thermograph. There is one half degree difference between each of the bars along the spectrum along the bottom of the illustration with relatively cool being light grey and relatively warm being dark grey. There is no more than one degree difference between any areas on the chest.

formula. Validity for each case was determined by taking the average of the coefficients while reliability was determined by taking the average of the correlations between each pair of raters.

\section{Results}

Differences between chest heat patterns of intact, incomplete SCI, and complete SCI subjects: Typical thermograms showing the chests of an intact subject, an incomplete SCI patient, and a complete SCI patient, are shown in Figures 1, 2 and 3 respectively. Figures 1 and 2 are grey tone renditions of coloured thermograms while Figure 3 was originally made in grey tone.

Colour versions of thermograms such as these were used by the panel in its attempt to distinguish between the three groups. Note that a temperature gradient band which crosses the chest of the complete SCI patient is absent in the intact and incomplete SCI people. The height of the band on the trunk appears to be generally related to the level of the injury. The transition zone is much less distinct among those complete SCI patients who reported a gradual transition in sensations. The average number of degrees difference between the area with normal sensations and the area with no or abnormal sensations was 1.5 degrees Celsius (standard deviation $(\mathrm{SD})=0.62$ ) for all of the complete SCI subjects and 0.58 ( $\mathrm{SD}=0 \cdot 29)$ for the incomplete SCI subjects. These are sig- 


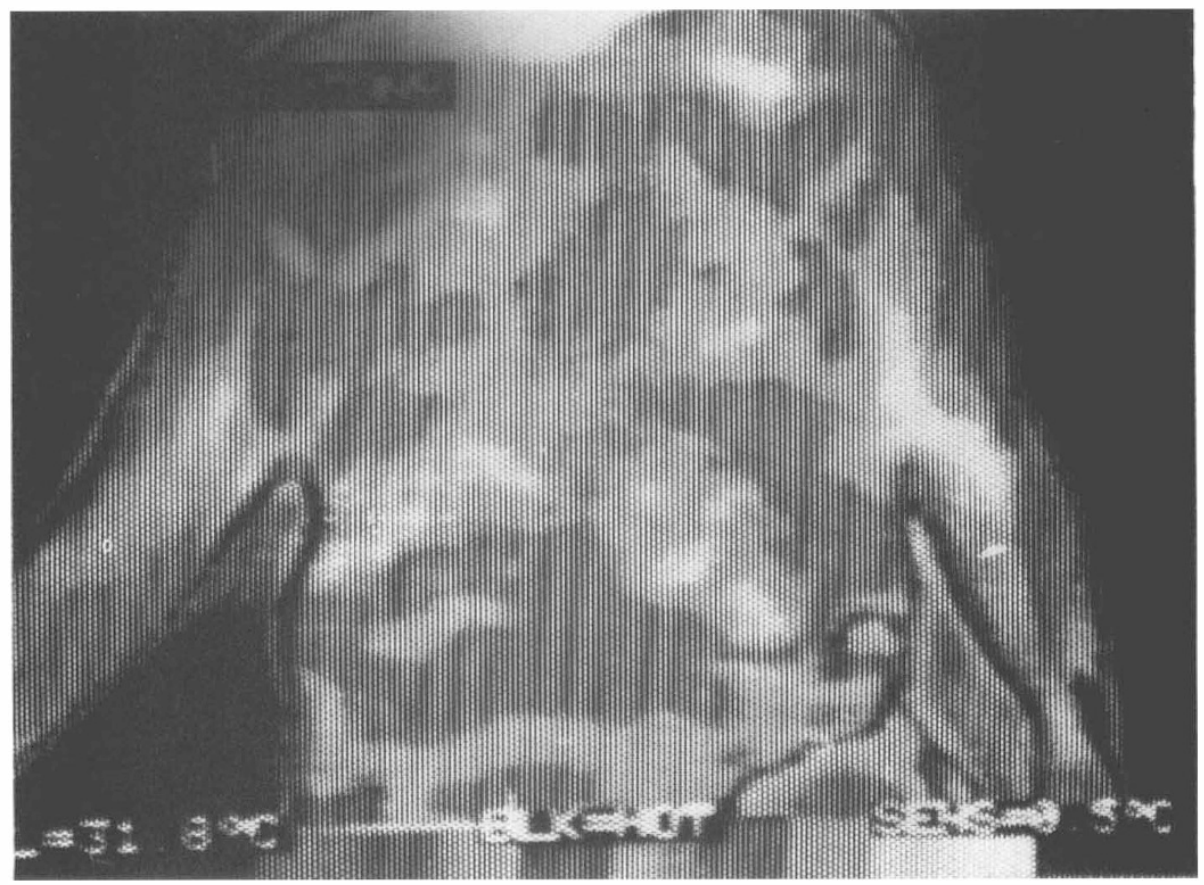

Figure 2 Is a typical thermogram of the chest of an incomplete spinal cord injured subject. The illustration is a greytone reproduction of a colour thermograph. There is one half degree difference between each of the bars along the spectrum along the bottom of the illustration with relatively cool being light grey and relatively warm being dark grey. There is no more than one degree difference between any areas on the chest. Minor differences between the thermogram of the intact subject and this illustration are due to random heat patterns rather than significant, replicable conditions.

nificantly different at $\mathrm{p}<0.01$. ( $\mathrm{t}=3.92$ with 21 degrees of freedom (DF)). There were no consistent differences between the patterns shown by intact and incomplete SCI subjects and differences were never greater than one degree. If the complete SCI group is divided between the ten reporting narrow, sharp zones and the five reporting gradual, long sensation transition zones, those with narrow zones show a mean of $1.5(\mathrm{SD}=0.57)$ while those with gradual changes a show a mean of $1.2(\mathrm{SD}=0 \cdot 84)$. When complete SCI patients having narrow zones are compared with incomplete SCI patients, they are significantly different at $\mathrm{p}<0.01(\mathrm{t}=4.09$ with $16 \mathrm{DF})$. The Table describes each subject's injury level, transition zone, thermal demarcation line, and results of the panel if that subject was included in the blind evaluation. Data from eight of the complete SCI subjects appeared in a previous article on pain in spinal cord injured veterans (Sherman et al., 1986b). In order to facilitate recognition of data from subjects appearing in both articles, we have kept the subject numbers for the original eight subjects the same as they were in the first article. We did not have trunk thermograms for two of the original subjects; so, their numbers are missing from the Table.

The blind panel was given chest or back thermograms of five of the complete SCI patients (three with sharp and two with gradual demarcations), all seven of 


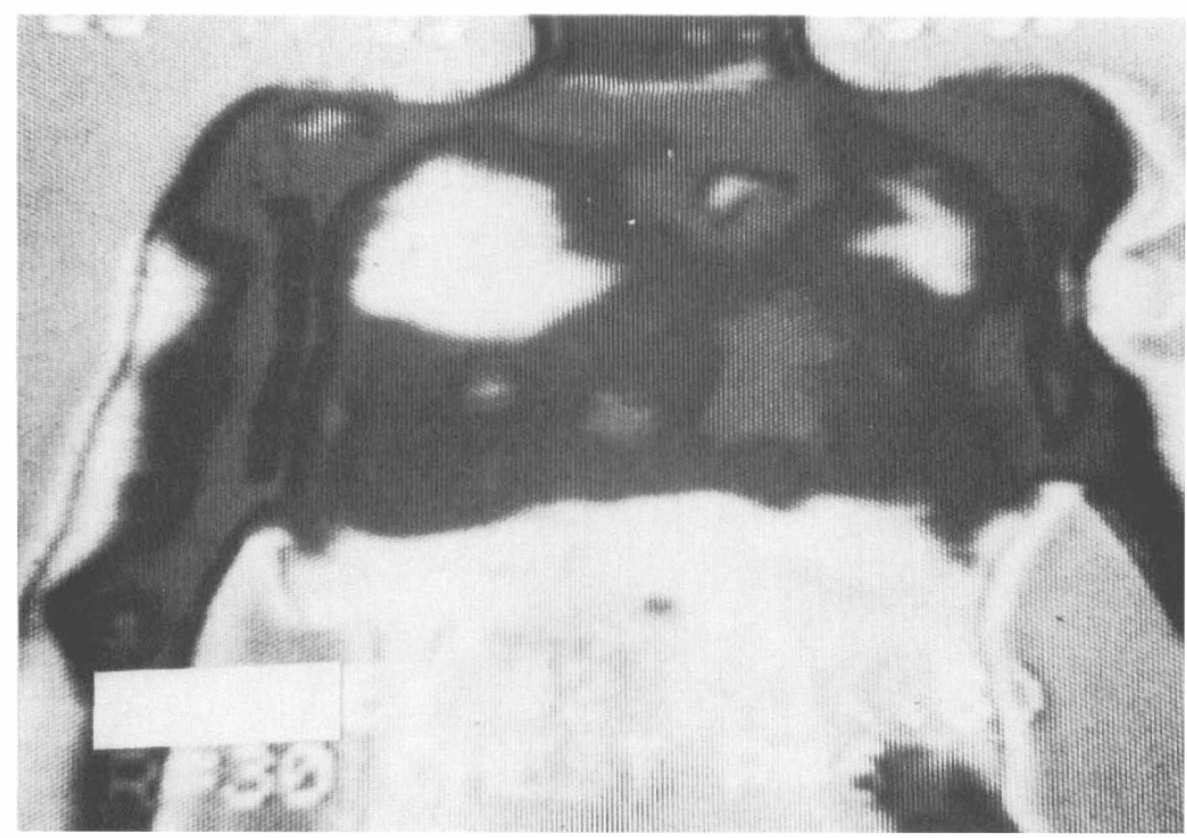

Figure 3 Is typical of thermograms showing the chest of a complete spinal cord injured subject. The illustration is a greytone thermograph having a continuum of darkness with light grey indicating relatively cool and dark grey indicating relatively warm. There is a minimum of $31 / 2$ degree Celsius difference between the lightest and darkest tones.

the incomplete SCI patients, and five intact subjects. All of the complete SCI subjects were not presented because this evaluation was part of the larger study discussed in the introduction. The number of photos had to be held to a minimum to keep each panelist's participation time to a length which would not preclude getting volunteers to participate in the panel. The panel had difficulty sorting only one of the complete and one of the incomplete SCI subjects. One of the complete SCI subjects (column 3 in the Table) had a very gradual transition involving only one-half degree. Only three panelists felt that this was a complete SCI case. One of the incomplete SCI subjects (column 24 in the Table) had a very diffuse pattern covering most of the chest which was partially caused by the distribution of chest hair. This anomaly confused half of the panel. The mean validity for the panel's attempt to distinguish complete from incomplete SCI subjects was 0.67 and their mean interrater reliability was 0.63 . One of the authors, who was not blind to the study but who did not know which thermograms related to individual subjects, sorted the thermograms for all subjects participating in the study. He correctly sorted all of the thermographs except the one related to the same complete injury subject (column 3 ) missed by the blind panel. None of the members of the panel or the author who reviewed the thermograms was able to distinguish between incomplete SCI and intact subjects. In fact, the mean validity for the panel was only -0.09 and the mean interrater reliability was $0 \cdot 01$.

Stability of patterns. We had the opportunity to record two of the complete and one of the incomplete SCI subjects several times. Their patterns appeared 
identical each time. Although the actual temperature of their bodies changed from recording to recording, the temperature difference between the upper and lower portions of the trunk remained constant.

\section{Discussion}

The possibility that the many interrelated mechanisms involved in production of trunk heat patterns function differently among complete and incomplete SCI people is of considerable theoretical and clinical interest. However, objective verification of our findings presents a very real problem because there is still no 'gold standard' against which the results of our study can be measured (e.g. Hussey, R., 1986, personal communication). A wide variety of nerve stimulation and conduction studies being conducted in various laboratories are close to producing objective ways to demonstrate the completeness of both motor and sensory lesions. When these tests are developed, they will still have to be compared to a group of people whose lesions are known to be complete in order to verify their accuracy. A direct approach to verification of our results would be to take thermograms of SCI subjects where cords were surgically severed for pain or other problems. If they show the same results as our 'complete' SCI subjects, the technique could be verified without waiting for the development of a 'gold standard.'

We were impressed by the ability of 'blind' raters, entirely naïve to evaluating thermograms, to quite consistently distinguish between incomplete and complete SCI subjects. We suspect that the 'complete' case which was not sorted correctly may represent an incomplete injury because there was only a small temperature difference between the normal and abnormal areas of the trunk.

If the technique proves to be as specific as initial results indicate, it will be of great value for quick, non-invasive evaluation of SCI patients coming to a SCI unit. Once the likely temperature range for the trunks of SCI subjects has been established, relatively inexpensive, easy to use contact thermographs can be substituted for the expensive videothermograph used in this study.

The opinions or assertions contained herein are the private views of the authors and are not to be construed as official or as reflecting the views of the United States Department of the Army, the Department of Defense, or the Veterans Administration.

\section{Acknowledgements}

This study was entirely supported by the Department of Clinical Investigation at Eisenhower Army Medical Center and the Rehabilitation Research Service of the US Veterans Administration. We acknowledge the help of the following scientists who comprised the blind review panel: Barry $\mathrm{N}$. Blum, Michael K. Drakeford, Leroy R. Fullerton Jr., Jacob A. Goodrich, Jack A. Horner, Robert C. Hulsebus, Rodney A. Sullivan, Federico M. Tamayo, Lawrence R. Walker, and Timothy R. Young. We appreciate the review of this article done by Dr. Kent M. Plowman of Eisenhower Army Medical Center. We also appreciate the help of Mr. Harry Davis of the Medical College of Georgia in analysing the data from the 'blind' portion of this study.

\section{References}

Guttmann L 1976 Spinal cord injuries-Comprehensive Management and Research. 2nd ed. pp. 314-330. Blackwell Scientific Publishers: Oxford.

Harway R 1986 Precision Thermal imaging of the extremities. Orthopedics 9:379-382. 
NORMELL L 1974 Recording of normal and impaired cutaneous thermoregulatory vasomotor responses by infra-red thermography: a methodological study. Scandinavian fournal of Clinical Laboratory Investigation 33(138):3-24.

SHERMAN R, BARJA R, BRUNO G 1986 Thermographic correlates of chronic pain: analysis of 125 sequential subjects incorporating evaluations by a blind panel. Archives of Physical Medicine and Rehabilitation, in the press.

SHERMAN R, ERNST J, MARKOWSKI J 1986 Relationships between near surface blood flow and altered sensations among spinal cord injured veterans. American fournal Physical Medicine, in press.

Trieschmann R 1980 Spinal cord injuries: Psychological, social, and vocational adjustment. Pergamon Press, New York. 